\title{
Phenolic Compounds and Cytotoxic Activities of Methanol Extract of Basil (Ocimum basilicum L.)
}

\author{
Mohamed HM Abd El-Azim ${ }^{1 *}$, Ahmed AM Abdelgawad ${ }^{2,3}$, MohamedEl-Gerby ${ }^{1}$, Sherin Ali ${ }^{1}$ and Amani MD El-Mesallamy ${ }^{1}$ \\ ${ }^{1}$ Department of Chemistry, Faculty of Science, Zagazig University, Zagazig, Egypt \\ ${ }^{2}$ Medicinal and Aromatic Plants Department, Desert Research Center, Cairo, Egypt \\ ${ }^{3}$ Department of Chemistry, Faculty of Science, Jazan University, Jizan, Saudi Arabia
}

\begin{abstract}
Chemical investigation of methanol extract of Ocimum basilicum L. resulted the isolation and identification of twelve phenolic compounds as $p$-hydroxy benzoic acid, ferulic acid, gallic acid, p-qumaric acid, benzoic acid, kaempferol, catechin, quercetin, chlorogenic acid, caffeic acid, cinnamic acid and ellagic acid. The structures of these compounds were confirmed by Chromatographic, UV, MS, ${ }^{1} \mathrm{H}-\mathrm{NMR}$ and ${ }^{13} \mathrm{C}-\mathrm{NMR}$ spectral data. The cytotoxic activity of the methanol extract of Ocimum basilicum showed strong cytotoxic activity against colon (HCT116) and liver (HEPG2) carcinoma cell line, where $\mathrm{IC}_{50}$ of the two human cell line were $27 \mu \mathrm{g} / \mathrm{ml}$ and $34.5 \mu \mathrm{g} / \mathrm{ml}$, respectively.
\end{abstract}

Keywords: Ocimum basilicum L.; Phenolic constituents; HCT116; HEPG2; Cytotoxic activity

\section{Introduction}

The Ocimum basilicum L. (basil) is an annual, herbaceous, white to purple flowering plant, $20-60 \mathrm{~cm}$ tall, that originated in Iran and India $[1,2]$. The genus Ocimum belonging to the Lamiaceae comprises annual and perennial herbs and shrubs native to the tropical and subtropical regions of Asia, Africa and South America [3]. The taxonomy of Ocimum is complex due to interspecific hybridization and polyploidy of the species in the genus. Pushpangadan and Bradu, [4] recognized more than 150 species; however, also Paton [5]; proposed that Ocimum had only 65 species and other attributions should be considered as synonyms. Basil is used as a medicinal herb in medical treatments such as for headaches, coughs, diarrhea, worms and kidney malfunctions. Basil essential oil has been utilized extensively in the food industry as a flavoring agent, and in perfumery and medical industries [6]. Basil (Ocimum basilicum L.) is an aromatic herb that is used extensively to add a distinctive aroma and flavor to food. The leaves can be used fresh or dried for use as a spice. Basil extract has antimicrobial and antioxidant activities due to its phenolic and aromatic compounds $[7,8]$. The main phenolics reported in basil are phenolic acids and flavonolglycosides $[9,10]$. Also, basil possesses insecticidal [11] properties and it is a promising fungi static [12]. Rosmarinic acid is the most prevalent basil phenolic, [13,14] but other caffeic acid derivatives, such as chicoric acid found in substantial concentrations [14]. In continuation of our studies, [15-17] we report here the chemical composition and antitumor activity of methanol extract of Egyptian Ocimum basilicum L., from the family Lamiaceae.

\section{Materials and Methods}

\section{Plant materials}

Ocimum basilicum L. was collected Aswan city (South Egypt), during spring season, 2012. The collected plants were identified by Botany Department, Faculty of Science, Zagazig University and by comparison with plant description in flora of Egypt. The plant parts were dried under shade and then grinded to fine powder.

\section{Chromatographic analysis}

Sheets of Whatman paper No 1 or $3 \mathrm{MM}$ were used for twodimensional, comparative or preparative paper chromatography. The separation of the phenolic and flavonoid components was performed by column fractionation of the extract or its fractions on one of the following stationary phases as stated in each case. A- Polyamide powder, polyamide 6-S for CC, Riedel-De Haen AG, seelze-Hannover, Germany. B-Sephadex LH-20, (25-100 $\mu \mathrm{m})$, Pharmacia fine chemicals.

\section{Spectroscopic analysis}

V spectroscopic: Investigated material and UV measurements were then carried out. Chromatographically, pure materials dissolved in analytically pure methanol were subjected to UV spectrophotometric investigation in $4 \mathrm{ml}$ capacity quartz cells Zeiss spectrometer PMQ-II. In case of flavonoids, $\mathrm{AlCl}_{3}, \mathrm{AlCl}_{3} / \mathrm{HCl}$, fused $\mathrm{NaOAc} / \mathrm{H}_{3} \mathrm{BO}_{3}$ and $\mathrm{NaOMe}$ reagents were separately added to methanolic solution of the investigated material and UV measurements were then carried out [18].

NMR spectroscopic: ${ }^{1} \mathrm{H}$ and ${ }^{13} \mathrm{C}$ chemical shifts $(\delta)$ were measured in ppm, relative to DMSO - d6 and converted to TMS scale. Jeol EC A $500 \mathrm{MHz}$ NMR Spectrometer at $500 \mathrm{MHz}$, (Institute Fur Chemie, Humboldt Universität zu Berlin, Germany). ${ }^{1} \mathrm{H}$ chemical shifts were measured in ppm, relative to TMS and 13C NMR chemical shifts to DMSO-d6 and converted to TMS scale by adding 39.5. Typical conditions: spectral width $=8 \mathrm{KHz}$ for $1 \mathrm{H}$ and $30 \mathrm{KHz}$ for $13^{\circ} \mathrm{C}, 64 \mathrm{~K}$ data points and a flip angle of $45^{\circ} \mathrm{C}$.

Mass spectroscopic: The isolation of pure compounds were subjected (FAB -MS). The isolated pure compounds were subjected, in most cases to Fast Atom Bombardment (positive and negative) mass spectroscopic analysis (FAB-MS) on MM 7070 E spectrometer (VG analytical). Some other compounds were subjected to electron spray ionization mass spectroscopic analysis (ESI-MS) a Varian Mat1 12-

*Corresponding author: Mohamed H M Abd El-Azim, Department of Chemistry, Faculty of Science, Zagazig University, Zagazig, Egypt, Tel: 00201067653545; E-mail: mhmsm01213@yahoo.com

Received May 18, 2015; Accepted June 13, 2015; Published June 20, 2015

Citation: Abd El-Azim MHM, Abdelgawad AAM, El-Gerby M, Ali S, El-Mesallamy AMD (2015) Phenolic Compounds and Cytotoxic Activities of Methanol Extract of Basil (Ocimum basilicum L.). J Microb Biochem Technol 7:4 182-185. doi:10.4172/1948-5948.1000202

Copyright: @ 2015 Abd El-Azim MHM, et al. This is an open-access article distributed under the terms of the Creative Commons Attribution License, which permits unrestricted use, distribution, and reproduction in any medium, provided the original author and source are credited. 
Citation: Abd El-Azim MHM, Abdelgawad AAM, El-Gerby M, Ali S, El-Mesallamy AMD (2015) Phenolic Compounds and Cytotoxic Activities of Methanol Extract of Basil (Ocimum basilicum L.). J Microb Biochem Technol 7:4 182-185. doi:10.4172/1948-5948.1000202

ET Spectrometer. All measurements were carried out at Institute Fur Chemie, Humboldt Universitat zu Berlin, Germany.

Extraction and isolation of the phenolic compounds: The dried plant (all parts of the plant), was extracted exhaustively with petroleum ether, diethyl ether and methanol (according to polarity). For each extraction, the powder was left $24 \mathrm{~h}$, in a Soxhlet apparatus. After that the methanol extract was concentrated under vacuum and left overnight. This yielded a gummy solid which was separated by filtration. The methanol filtration was shown by paper chromatography used twodimensional (TDPC), comparative or preparative paper chromatography to determine phenolic acid and flavonoids compounds [16].

$\rho$-Hydroxy benzoic acid: $\mathrm{R}_{\mathrm{f}}$ values (x 100): 36(HOAc-6), 84(BAW). $\mathrm{UV} / \mathrm{Vis}\left(\mathrm{MeOH}, \lambda_{\max }, \mathrm{nm}\right): 253 .{ }^{1} \mathrm{H}-\mathrm{NMR}\left(400 \mathrm{MHz}, \mathrm{DMSO}-d_{6}, \delta\right.$, ppm): $7.8(d, j=9 \mathrm{~Hz})$ and $6.8(d, j=9 \mathrm{~Hz})$.

Ferulic acid: $\mathrm{R}_{\mathrm{f}}$ values (x100): 40( $\left.\mathrm{H}_{2} \mathrm{O}\right), 42(\mathrm{HOAC}-6 \%), 86(\mathrm{BAW})$. UV UV/Vis (MeOH, $\left.\lambda_{\max }, \mathrm{nm}\right): 268$, 313. ${ }^{1} \mathrm{H}-\mathrm{NMR}(400 \mathrm{MHz}$, DMSO- $\left.d_{6}, \delta, \mathrm{ppm}\right): 3.81\left(s, \mathrm{CH}_{3}\right), 6.69(d, \mathrm{H}-\alpha), 6.18(d, \mathrm{H}-5), 7.10(d$, $\mathrm{H}-6), 7.30(s, \mathrm{H}-2), 7.52(d, \mathrm{H}-\beta)$.

Gallic acid: $\mathrm{R}_{\mathrm{f}}$ values (x100): 44( $\left.\mathrm{H}_{2} \mathrm{O}\right), 55(\mathrm{HOAc}-6), 72(\mathrm{BAW})$. UV/Vis $\left(\mathrm{MeOH}, \lambda_{\max }, \mathrm{nm}\right): 272 .{ }^{1} \mathrm{H}-\mathrm{NMR}\left(400 \mathrm{MHz}, \mathrm{DMSO}-d_{\sigma}, \delta\right.$, ppm): $6.98\left(s, 2 \mathrm{H}, \mathrm{H}-2\right.$ and H-6). ${ }^{13} \mathrm{C}-\mathrm{NMR}\left(400 \mathrm{MHz}, \mathrm{DMSO}-d_{6}, \delta\right.$, ppm): 120.6(C-1), 108.8(C-2 and C-6), 145.5(C-3 and C-5), 138.1(C4), 167.7(C=O). MS (EI, $m / z(\%)): 170.9\left[\mathrm{M}^{+}+1,5.1\right], 169.0\left[\mathrm{M}^{+}-\mathrm{H}\right.$, 100, ion $\mathrm{A}], 167.9\left[\mathrm{M}^{+}-\mathrm{H}_{2}, 63.9\right], 153\left[\mathrm{M}^{+}-\mathrm{OH}, 11.2\right], 145\left[\right.$ ion $\mathrm{A}-\mathrm{C}_{2}$, 3.5], 139 [ion $\mathrm{A}-\mathrm{HCHO}, 11.3$ ], $126\left[\mathrm{M}^{+}-\mathrm{CO}_{2}, 6.5\right.$ ] and 123 [ion $\mathrm{A}-$ $\mathrm{HCOOH}, 27.1$ ]. Anal. Calcd for $\mathrm{C}_{7} \mathrm{H}_{6} \mathrm{O}_{5}$ (170.02): C, 49.42; H, 3.55; O, 47.02. Found: C, 49.50; H, 3.30; O, 47.20.

$\rho$-Coumaric acid: $\mathrm{R}_{\mathrm{f}}$ values (x100): 43( $\left.\mathrm{H}_{2} \mathrm{O}\right), 45(\mathrm{HOAC}-6 \%)$, 90(BAW). UV/Vis $\left(\mathrm{MeOH}, \lambda_{\max }, \mathrm{nm}\right): 266,310 ;(\mathrm{MeOH}+\mathrm{NaOMe}$, $\left.\lambda_{\max }, \mathrm{nm}\right): 228,333 .{ }^{1} \mathrm{H}-\mathrm{NMR}\left(400 \mathrm{MHz}, \mathrm{DMSO}-d_{6}, \delta, \mathrm{ppm}\right): 6.2(d$, $J=15 \mathrm{~Hz}, \mathrm{H}-\alpha), 6.72(d, J=8 \mathrm{~Hz}, \mathrm{H}-3$ and $\mathrm{H}-5), 7.32(d, J=8 \mathrm{~Hz}, \mathrm{H}-2$ and $\mathrm{H}-6), 7.52(d, J=15 \mathrm{~Hz}, \mathrm{H}-\beta)$. Anal. Calcd for $\mathrm{C}_{9} \mathrm{H}_{8} \mathrm{O}_{3}$ (164.16): C, 65.85; $\mathrm{H}, 4.91 ; \mathrm{O}, 29.24$. Found: C, 65.80; H, 4.80; O, 29.40.

Benzoic acid: ${ }^{1} \mathrm{H}-\mathrm{NMR}\left(400 \mathrm{MHz}, \mathrm{DMSO}-d_{6}, \delta, \mathrm{ppm}\right): 12.9(s$, $\left.\mathrm{H}_{\text {Carboxylic }}\right), 8.20(d, \mathrm{H}-2$ and $\mathrm{H}-6), 7.83(m, \mathrm{H}-3$ and $\mathrm{H}-5)$ and $7.4(m, \mathrm{H}-4)$.

Kaempherol: $\mathrm{R}_{\mathrm{f}}$ values (x100): 00( $\left.\mathrm{H}_{2} \mathrm{O}\right), 10(\mathrm{HOAc}-6), 85(\mathrm{BAW}) . \mathrm{UV} /$ Vis $\left(\mathrm{MeOH}, \lambda_{\text {max }}, \mathrm{nm}\right): 268,369 ;\left(\mathrm{MeOH}+\mathrm{NaOAc}, \lambda_{\max }, \mathrm{nm}\right): 270,310$, 375; $\left(\mathrm{NaOAc}+\mathrm{H}_{3} \mathrm{BO}_{3}, \lambda_{\max }, \mathrm{nm}\right): 270,320,372 ;\left(\mathrm{MeOH}+\mathrm{AlCl}_{3}, \lambda_{\max }\right.$, $\mathrm{nm}): 270,305,360,430 ;\left(\mathrm{AlCl}_{3}+\mathrm{HCl}, \lambda_{\max }, \mathrm{nm}\right): 278,316,413 .{ }^{1} \mathrm{H}-\mathrm{NMR}$ (400 MHz, DMSO- $d, \delta$, ppm): $6.4(d, J=2.5, \mathrm{H}-8), 6.18(d, J=2.5, \mathrm{H}-6)$, $8.14\left(d, J=8, \mathrm{H}-2^{\prime}\right.$ and $\left.\mathrm{H}-6^{\prime}\right), 6.89\left(d, J=8, \mathrm{H}-3^{\prime}\right.$ and $\left.\mathrm{H}-5^{\prime}\right) .{ }^{13} \mathrm{C}-\mathrm{NMR}(400$ MHz, DMSO- $\left.d_{6}, \delta, \mathrm{ppm}\right): 146.8(\mathrm{C}-2), 135.4(\mathrm{C}-3), 175.9(\mathrm{C}-4), 161.0(\mathrm{C}-$ 5), 98.6(C-6), 164.2(C-7), 93.8(C-8), 156.4(C-9), 103.7(C-10), 121.9(C$\left.1^{\prime}\right), 129.9\left(\mathrm{C}-2^{\prime}\right.$ and $\left.\mathrm{C}-6^{\prime}\right), 115.8\left(\mathrm{C}-3^{\prime}\right.$ and C-5') and 159.5(C-4'). MS (EI, $m / z(\%)): 285.1\left[\mathrm{M}^{-}-\mathrm{H}, 100\right.$, ion $\mathrm{A}$ ], $242.1\left[\mathrm{M}^{-}-\mathrm{CO}_{2}, 5.3\right.$ ], 203.1 [ion $\mathrm{A}-\mathrm{C}_{4} \mathrm{H}_{2} \mathrm{O}_{2}, 4.6$, ion B] and 169.1[ion B $-\mathrm{H}_{2} \mathrm{O}_{2}, 5.7$ ].

Catechin acid: $\mathrm{R}_{\mathrm{f}}$ values ( $\mathrm{x}$ 100): 0.33( $\left.\mathrm{H}_{2} \mathrm{O}\right), 0.54(\mathrm{HOAC}-6)$, 0.60 (BAW). UV/Vis $\left(\mathrm{MeOH}, \lambda_{\max }, \mathrm{nm}\right): 278 .{ }^{1} \mathrm{H}-\mathrm{NMR}(400 \mathrm{MHz}$, DMSO- $\left.d_{6}, \delta, \mathrm{ppm}\right): 4.51(d, J=7.3, \mathrm{H}-2), 3.84(m, \mathrm{H}-3) 2.38$ (ax, $d d$, $J=16.0,7.9, \mathrm{H}-4), 2.68$ (eq, $d d, J=16.0,5.3, \mathrm{H}-4), 5.90(d, J=2.2, \mathrm{H}-6)$, $5.72(d, J=2.2, \mathrm{H}-8), 6.74\left(d, J=1.9, \mathrm{H}-2{ }^{\prime}\right), 6.7\left(d, J=8, \mathrm{H}-5^{\prime}\right), 6.61(d d$, $J=8,1.9, \mathrm{H}-6$ '). ${ }^{13} \mathrm{C}-\mathrm{NMR}\left(400 \mathrm{MHz}, \mathrm{DMSO}-d_{6}, \delta, \mathrm{ppm}\right): 81.0(\mathrm{C}-2)$, 66.4(C-3), 27.7(C-4), 156.1(C-5), 95.3(C-6), 156.4(C-7), 94.0(C-8), 155.3(C-9), 99.2(C-10), 130.7(C- 1'), 114.5(C-2'), 144.8(C-3'), 144.8(C4'), 115.1(C-5'), 118.4(C-6').

Quercetin: $\mathrm{R}_{\mathrm{f}}$ values (x 100): 00( $\left.\mathrm{H}_{2} \mathrm{O}\right), 07(\mathrm{HOAc}-6), 75(\mathrm{BAW})$. UV/Vis
$\left(\mathrm{MeOH}, \lambda_{\max }, \mathrm{nm}\right): 255,268,370 ;\left(\mathrm{MeOH}+\mathrm{NaOAc}, \lambda_{\max }, \mathrm{nm}\right): 254,276$, 375; $\left(\mathrm{NaOAc}+\mathrm{H}_{3} \mathrm{BO}_{3}, \lambda_{\max }, \mathrm{nm}\right): 272,388 ;\left(\mathrm{MeOH}+\mathrm{AlCl}_{3}, \lambda_{\max }, \mathrm{nm}\right)$ : 270, 360, 440; $\left(\mathrm{AlCl}_{3}+\mathrm{HCl}, \lambda_{\max }, \mathrm{nm}\right): 258,400 .{ }^{1} \mathrm{H}-\mathrm{NMR}(400 \mathrm{MHz}$ DMSO- $d, \delta$, ppm): $6.19(d, J=2.5, \mathrm{H}-6), 6.4(d, J=2.5, \mathrm{H}-8), 7.64(d$, $\left.J=2.5, \mathrm{H}-2^{\prime}\right), 6.88\left(\mathrm{~d}, \mathrm{~J}=8.5, \mathrm{H}-5^{\prime}\right), 7.53\left(d d, J=2.5\right.$ and $\left.8.5, \mathrm{H}-6{ }^{\prime}\right) .{ }^{13} \mathrm{C}-$ NMR (400 MHz, DMSO- $d_{6}, \delta$, ppm): 147.0(C-2), 135.8(C-3), 176.2(C4), 160.5(C-5), 99.2(C-6), 164.0(C-7), 93.7(C-8), 156.4(C-9), 103.5(C10), 122.2(C-1'), 115.3(C-2'), 145.1(C-3'), 148.0(C-4'), 115.6(C-5') and 120.2(C-6'). MS (EI, $m / z(\%)): 300.8$ [M- $-\mathrm{H}, 100$, ion A], 299.8 [ $\mathrm{M}^{-}-\mathrm{H}_{2}$, 28.3], 270.9 [ion $\mathrm{A}-\mathrm{C}_{2} \mathrm{H}_{6}, 8.9$ ], 162.9 [ion $\mathrm{A}-\mathrm{C}_{7} \mathrm{H}_{6} \mathrm{O}_{3}, 10.1$, ion $\mathrm{B}$ ] and 117.3 [ion $\mathrm{B}-\mathrm{HCOOH}, 5.3$ ]. Anal. Calcd. for $\mathrm{C}_{15} \mathrm{H}_{10} \mathrm{O}_{7}$ (302.04): C, 59.61; H, 3.33; O, 37.06. Found: C, 59.30; H, 3.40; O, 37.30.

Chlorogenic acid: $\mathrm{R}_{\mathrm{f}}$ values (x100): $67\left(\mathrm{H}_{2} \mathrm{O}\right), 65$ (HOAC-6\%), 59 (BAW). UV/Vis $\left(\mathrm{MeOH}, \lambda_{\max }, \mathrm{nm}\right): 220,245,300,330 ;(\mathrm{MeOH}+$ NaOMe, $\left.\lambda_{\max }, \mathrm{nm}\right): 229,275,400 .{ }^{1} \mathrm{H}-\mathrm{NMR}\left(400 \mathrm{MHz}, \mathrm{DMSO}-d_{6}, \delta\right.$, ppm): Caffeic acid moiety : $7.42(d, J=16 \mathrm{~Hz}, \beta-\mathrm{H}), \quad 7.05(d, J=2 \mathrm{~Hz}$, $6-\mathrm{H}), 6.96(d d, J=7.5 \mathrm{~Hz}$ and $J=2 \mathrm{~Hz}, 2-\mathrm{H}), 6.79(d, J=7.5 \mathrm{~Hz}, 3-\mathrm{H})$, $6.19(d, J=16 \mathrm{~Hz}, \alpha-\mathrm{H})$; Quinic acid moiety: $5\left(m, 1^{\prime}-\mathrm{H}\right), 1.88(m$, $2^{\prime}-\mathrm{H}$ and $\left.6^{\prime}-\mathrm{H}\right), 3.85\left(m, 3^{\prime}-\mathrm{H}\right.$ and $\left.5^{\prime}-\mathrm{H}\right), 3.5\left(m, 4{ }^{\prime}-\mathrm{H}\right) .{ }^{13} \mathrm{C}$ NMR $(400$ $\left.\mathrm{MHz}, \mathrm{DMSO}-d_{6}, \delta, \mathrm{ppm}\right)$ : Caffeic acid moity:126.1(C-1), 115.2(C-2), 144.9(C-3), 148.5(C-4), 116.29(C-5), 121.5(C-6), 146.2(C-7), 115.2(C7), 115.2(C-8),116.5(C-9); Quinic acid moiety: 76.6(C-1'), 68.6(C-3'), 71.8(C-4'), 71(C-5'), 180(C-7').

Caffeic acid: $\mathrm{R}_{\mathrm{f}}$ values (x 100): 25( $\left.\mathrm{H}_{2} \mathrm{O}\right), 45(\mathrm{HOAC}-6 \%), 81$ (BAW). UV/Vis $\left(\mathrm{MeOH}, \lambda_{\max }, \mathrm{nm}\right): 218,245,298,325 .{ }^{1} \mathrm{H}-\mathrm{NMR}(400 \mathrm{MHz}$, DMSO- $d, \delta, p p m): 6.2(d, J=16 \mathrm{~Hz}, \beta-\mathrm{H}), 6.76(J=7.5 \mathrm{~Hz}, 5-\mathrm{H}), 6.88(d d$, $J=7.5 \mathrm{~Hz}$ and $J=2.5 \mathrm{~Hz}, 6-\mathrm{H}), 6.98(d, J=2.5 \mathrm{~Hz}, 2-\mathrm{H}), 7.48(d, J=16 \mathrm{~Hz}$, $\alpha-\mathrm{H})$.

Cinnamic acid: $\mathrm{R}_{\mathrm{f}}$ values (x100): 30( $\left.\mathrm{H}_{2} \mathrm{O}\right), 41(\mathrm{HOAC}-6 \%)$, 80(BAW). ${ }^{1} \mathrm{H}-\mathrm{NMR}(400 \mathrm{MHz}, \mathrm{DMSO}-d, \delta, \mathrm{ppm}): 6.46$ ( $\left.d, \mathrm{H}-\alpha\right), 7.40$ $(t, \mathrm{H}-3$ and $\mathrm{H}-5), 7.42(t, \mathrm{H}-4), 7.557$ (d, H-2 and H- 6), 7.806 (d, H- $\beta$ and C-6'). ${ }^{13} \mathrm{C}$ NMR (400 MHz, DMSO- $\left.d_{6}, \delta, \mathrm{ppm}\right): 159.2(\mathrm{C}-7$ and C-7').

Ellagic acid: $\mathrm{R}_{\mathrm{f}}$ values (x 100): $0\left(\mathrm{H}_{2} \mathrm{O}\right), 9(\mathrm{HOAc}-6), 48(\mathrm{BAW}) . \mathrm{UV} /$ Vis $\left(\mathrm{MeOH}, \lambda_{\max }, \mathrm{nm}\right): 255,362 .{ }^{1} \mathrm{H}-\mathrm{NMR}\left(400 \mathrm{MHz}, \mathrm{DMSO}-d_{6}, \delta\right.$, ppm): 7.48 ( $s, \mathrm{H}-5$ and H-5'). ${ }^{13} \mathrm{C}$ NMR (400 MHz, DMSO- $\left.d_{6}, \delta, \mathrm{ppm}\right)$ : 112.3(C-1 and C-1'), 136.4(C-2 and C-2'), 140.2(C-3 and C-3'), 153(C-4 and $\left.\mathrm{C}-4^{\prime}\right), 111.4\left(\mathrm{C}-5\right.$ and $\left.\mathrm{C}-5^{\prime}\right), 107.6\left(\mathrm{C}-6^{\prime}\right)$.

\section{SRB assay of cytotoxic activity}

Measurement of potential cytotoxic activity of methanol extract of O. basilicum against the liver (HEPG2) and colon carcinoma cell line (HCT116) was tested by SRB (Sulphorhodamine-B) assay according to the method of Skehan et al. [19]. Human tumor cell lines were obtained frozen in liquid nitrogen $\left(-180^{\circ} \mathrm{C}\right)$ from the American Type Culture Collection. The tumor cell lines were maintained in The National Cancer Institute, Cairo, Egypt, by serial sub-culturing. This experiment was conducted in the National Cancer Institute, Cairo, Egypt.

\section{Results and Discussion}

\section{Results of phenolic constituents in methanol extract of $O$. basilicum}

Investigation of the phenolic compounds was done by fractionation of the extract, over polyamide column and elution with methanol/bidistilled water mixtures of decreasing polarities for gradient elution led to the desorption of sub fractions which were dried, individually, in vacuum, and then subjected to re-chromatography for several times led to the separation of twelve pure phenolic compounds. The structure 
Citation: Abd El-Azim MHM, Abdelgawad AAM, El-Gerby M, Ali S, El-Mesallamy AMD (2015) Phenolic Compounds and Cytotoxic Activities of Methanol Extract of Basil (Ocimum basilicum L.). J Microb Biochem Technol 7:4 182-185. doi:10.4172/1948-5948.1000202

of these compounds was confirmed by comparison of their physical and spectral data with those of reported compounds. Compounds were identified as $p$-hydroxy benzoic acid, ferulic acid, gallic acid, $p$-qumaric acid, benzoic acid, kaempferol, catechin, quercetin, chlorogenic acid, caffeic acid, cinnamic acid and ellagic acid.

\section{Anti-tumor activity}

The methanol extract of $O$. basilicum were tested against liver (HEPG2) and colon carcinoma cell lines (HCT116), the results showed the strong efficient cytotoxic activity of the methanol extract of $O$. basilicum against the tested human cell lines, the results were summarized in Figure 1,2. The $\mathrm{IC}_{50}$ values were calculated for colon and liver carcinoma cell lines as 34.5 and $27 \mu \mathrm{g} / \mathrm{ml}$, respectively.

\section{Conclusion}

The methanol extract of $O$. basilicum was found to include twelve active phenolic compounds. Also the extract was found to have strong efficient cytotoxic activity against liver (HEPG2) and colon carcinoma cell lines (HCT116).<smiles>[R]Oc1cc(C(=O)O)cc([R3])c1[R]</smiles>

12<smiles>[R]c1ccc(/C=C/C(=O)O)c([R])c1[R]</smiles>

$2 \quad \begin{array}{ll}\mathrm{R}_{1} \\ \mathrm{OCH}\end{array}$

$4 \mathrm{H}$

$10 \mathrm{H}$

$11 \mathrm{H}$
$\mathrm{OH} \quad \mathrm{H}$

$\mathrm{H} \quad \mathrm{OH}$

$\mathrm{OH} \mathrm{OH}$

$\mathrm{H} \quad \mathrm{H}$<smiles>[R]c1cc(-c2oc3cc(O)cc(O)c3c(=O)c2O)ccc1O</smiles>

6, $\mathrm{R}_{1}=\mathrm{H}$

$8, \mathrm{R}_{1}=\mathrm{OH}$

Figure 1: The chemical structure of the isolated phenolic compounds from the methanol extract of O. basilicum.

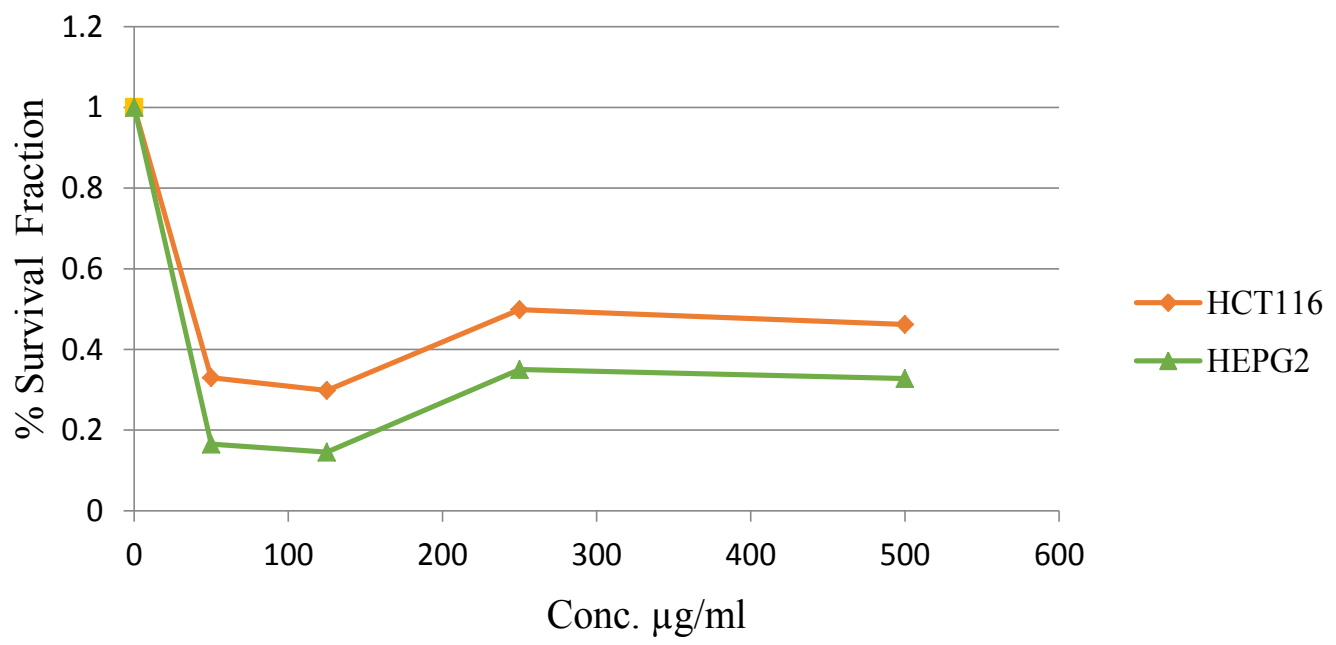

Figure 2: \% of survival fraction of colon and liver carcinoma cell lines against the concentration $(\mu \mathrm{g} / \mathrm{ml})$ of the methanol extract of $O$. basilicum. 
Citation: Abd El-Azim MHM, Abdelgawad AAM, El-Gerby M, Ali S, El-Mesallamy AMD (2015) Phenolic Compounds and Cytotoxic Activities of Methanol Extract of Basil (Ocimum basilicum L.). J Microb Biochem Technol 7:4 182-185. doi:10.4172/1948-5948.1000202

\section{References}

1. Chalchat JC, Ozcan MM (2008) Comparative essential oil composition of flowers, leaves and stems of basil (Ocimum basilicum $\mathrm{L}$ ) used as herb. Food Chem 110: 501-503.

2. Özcan M, Arslan D, Ünver A (2005) Effect of drying methods on the mineral content of basil (Ocimum basilicum L). Journal of Food Engineering 69: 375-379.

3. Darrah HH (1988) The Cultivated Basil. Buckeye Printing, Independence, MO

4. Pushpangadan P, Bradu BL (1995) Advances in Horticulture. Medicinal and Aromatic Plants, Malhotra Publishing House, New Delhi.

5. Paton A, Harley RM, Harley MM (1999) Ocimum overview of relationships and classification. Medicinal and Aromatic Plants-Industrial Profiles. Harwood Academic, Amsterdam.

6. Simon JE, Quinn J, Murray RG (1999) Basil: a source of essential oils. Advanced in New Crops. Timber Press, Portland.

7. Jayasinghe C, Gotoh N, Aoki T, Wada S (2003) Phenolics composition and antioxidant activity of sweet basil (Ocimum basilicum L). J Agric Food Chem 51: $4442-4449$

8. Hussain Al, Anwar F, Hussain Sherazi ST, Przybylski R (2008) Chemical composition, antioxidant and antimicrobial activities of basil (Ocimum basilicum) essential oils depends on seasonal variations. Food Chem 108: 986-995.

9. Lee J, Scagel CF (2009) Chicoric acid found in basil (Ocimum basilicum L.) leaves. Food Chemistry 115: 650-656.

10. Grayer RJ, Kite GC, Veitch NC, Eckert MR, Marin PD, et al. (2002) Lea flavonoid glycosides as chemo systematic characters in Ocimum. Biochemical Systematics and Ecology 30: 327-342.
11. Keita SM, Vincent C, Schmit JP, Belanger A (2000) Essential oil composition of Ocimum basilicum L., O. gratissimum L. and O. suave L. in the Republic of Guinea. Flavour and Fragrance Journal 15: 339-341.

12. Rai MK, Qureshi S, Pandey AK (1999) In vitro susceptibility of opportunistic Fusarium spp. to essential oils. Mycoses 42: 97-101.

13. Javanmardi J, Khalighi A, Kashi A, Bais HP, Vivanco JM (2002) Chemical characterization of basil (Ocimum basilicum L.) found in local accessions and used in traditional medicines in Iran. J Agric Food Chem 50: 5878-5883.

14. Lee J, Scagel CF (2009) Chicoric acid found in basil (Ocimum basilicum L.) leaves. Food Chemistry 115: 650-656.

15. Abd El Azim MHM, El-Mesallamy AMD, El-Gerby M, Awad A (2014) Anti-tumor antioxidant and antimicrobial and the phenolic constituents of Glycyrrhiza glabra. Organic Chemistry: An Indian Journal 10: 410-416.

16. Abd El Azim MHM, El-Mesallamy AMD, El-Gerby M, Awad A (2014) AntiTumor, Antioxidant and antimicrobial and the Phenolic Constituents of Clove Flower Buds (Syzygium aromaticum). Journal of Microbial and Biochemical Technology S8-007.

17. El-Mesallamy AMD, Hussein SAH, Abd El Azim MHM, El-Gerby M (2013) Phenolic composition and biological activities of methanolic extract of strawberry leaves (Fragaria ananassa). Natural products an Indian journal 9: 251-257.

18. Harborne JB, William CA (1975) "The Flaonoids" Chapman and Hall, London.

19. Skehan P, Storeng R, Scudiero D, Monks A, McMahon J, et al. (1990) New colorimetric cytotoxicity assay for anticancer-drug screening. J Natl Cancer Inst 82: 1107-1112. 